\title{
Prevalence and Antibiotic Susceptibility Pattern of Enterococcus spp. from Various Clinical Samples of Patients at a Tertiary Care Hospital, Patna
}

\author{
Shiwani Mishra, Richa Sinha*, Namrata Kumari, Kumar Saurabh, \\ Shailesh Kumar and S. K. Shahi \\ Department of Microbiology, Indira Gandhi Institute of Medical Sciences, Patna, India \\ *Corresponding author
}

\section{A B S T R A C T}

Keywords

Enterococci,

Antimicrobial

susceptibility,

Vancomycin

resistant

Enterococci, MDR, Bacterial infection

Article Info

\section{Accepted:}

12 March 2021

Available Online:

10 April 2021
Enterococci are opportunistic human pathogen and are gaining importance due to their high level of resistance against antimicrobials (betalactams, aminoglycosides, glycopeptides). The present study aims to estimate the burden of Enterococcal infection and determine its antibiogram at our multi-speciality hospital. This hospital based retrospective study was carried out in the Dept. of Microbiology, IGIMS, Patna. Isolates of Enterococci from various clinical samples were subjected to antibiotic susceptibility testing and speciation. Laboratory procedures were followed as per CLSI recommendation. From a total of 13,046 sample processed, 286 Enterococcus spp.were isolated, of which $234(83 \%)$ were from IPD and 52(17\%) from OPD patients. Maximum number of Enterococcus spp. were recovered from urine (246) followed by pus(16). Isolates were found to be resistant to ciprofloxacin (92\%), levofloxacin (85\%), and high-level gentamicin $(60 \%)$.Only one of the isolates showed resistance to linezolid. Sensitivity to vancomycin and nitrofurantoin (in urine) were $96 \%$ and $78 \%$ respectively. Antibiotic resistance is an ever-increasing problem which complicates management in patients with Enterococcal infection. Prudent use of antibiotics is a fundamental strategy to avoid such problems.

\section{Introduction}

Enterococci are ubiquitous gram positive, non-spore forming, facultative anaerobic cocci belonging to group D Streptococci as characterized by Lancefield in 1938, whose taxonomy has changed considerably in last few years (Parmeswarappa et al., 2013). They are part of normal intestinal flora in humans, but are also responsible for causing opportunistic infections (Murray et al., 2000). Earlier they were considered pathogen of low virulence but during the last three decades, their impact in nosocomial settings has gained our concern. They primarily affect debilitated and immunocompromised patients and exhibit resistance to many antimicrobials. Enterococci affects multiple anatomic sites and produce a 
variety of infections like bacteremia, endocarditis, UTIs, abdominal and pelvic infections, skin and soft tissue infections, bone and joints infections, CNS infections and pulmonary infections. In the Indian scenario, Enterococci are emerging as nosocomial pathogens which are difficult to treat. The clinical importance of Enterococci is directly related to its changing antibiogram. Prolonged stay in hospital, empirical use of antibiotics, lack of sufficient knowledge and poor implementation programs to control the rapid spread of Enterococci has led to increased mortality caused by these infections (Bhatt et al.,2015 \& Arias et al., 2012). Enterococci are regarded as "tough bugs" capable of withstanding many environmental stressors and surviving for long periods. They are tolerant of a wide range of environmental conditions, extreme temperature $\left(10^{0}-45^{0} \mathrm{C}\right)$, $\mathrm{pH}(4.5-10.0)$ and high sodium chloride concentrations, enabling them to colonize a wide range of niches (Arias et al.,2014). Till date, literature describes 12 Enterococcal species which are pathogenic to human, most common being Enterococcus faecalis and Enterococcus faecium.

Enterococcus spp. are intrinsically resistant to a number of antimicrobials including cephalosporins, trimethoprim sulfamethoxazole and also exhibit low level resistance to beta lactams and aminoglycosides (Mulla et al., 2012 \& Tripathi et al., 2016). This intrinsic resistance to several commonly used antimicrobials is the main reason for their survival despite aggressive treatment. Enterococci are also capable in acquiring resistance either through chromosome transfer of plasmid or by acquisition of transposons. Their capability of transferring determinants of antibioticresistant genes between the species, as well as other bacteria (Staphylococcus aureus), is a global cause of concern. Dramatic increase in antibiotic resistance of Enterococcus species worldwide highlights the need for greater understanding of this genus.

\section{Materials and Methods}

This retrospective study was carried out in the department of Microbiology, Indira Gandhi Institute of Medical Sciences(IGIMS), Patna, India. Aim was to determine the prevalence of Enterococci in various clinical samples of patients coming to the institute for treatment. All the clinical samples like blood, CSF, pleural fluid, peritoneal fluid, urine, wound swabs and pus swabs received from January 2018 to December 2018 showing growth of Enterococci in culture were included in the study.

Samples were processed and the pathogen identified as per routine laboratory protocol. Standard guidelines issued by Clinical Laboratories Standards Institute (CLSI) were used for testing the anti-microbial susceptibility pattern of all the isolates(CLSI 2016).

Samples were subjected to gram's staining and then cultured onto 5\%sheep blood agar, chocolate agar and MacConkey agar plates. Urine samples were also cultured on CLED plates. They were incubated for 18- 24 hours at $37^{0} \mathrm{C}$ and growth on culture plates were described. On sheep blood agar plate colonies were as $0.5-1 \mathrm{~mm}$ in diameter and nonhemolytic. On MacConkey agar they appeared as small dark-red magenta colored colonies.

Growth were subjected to Gram staining procedure, in which it showed gram positive cocci in pairs. Colonies were confirmed as Enterococci with the help of various biochemicals like negative catalase test, positive bile esculin test, growth in $6.5 \% \mathrm{NaCl}$ broth(Konemann's Color Atlas 2006). Further speciation was done on the basis of pigment production, motility test and standard 
biochemical reactions as per standard microbiological guidelines.

All Enterococcal isolates were then subjected to modified Kirby-Bauer antibiotic susceptibility test using standard techniques as per CLSI recommendations (Facklam et al., 1989 \& Murray et al.,). The panel of antibiotic discs that were used for susceptibility testing of Enterococcal isolates were ampicillin (10mcg), ciprofloxacin (5mcg), gentamicin high content $(120 \mathrm{mcg})$, vancomycin $(30 \mathrm{mcg})$ and linezolid $(30 \mathrm{mcg})$.

For urine isolates, an additional nitrofurantoin $(300 \mathrm{mcg})$ disc was used. With the help of a straight inoculating wire, 3-5 well-isolated colonies of the same morphology were picked and transferred into a tube containing $5 \mathrm{ml}$ of Mueller Hinton broth. The broth culture was incubated at $35^{\circ} \mathrm{C}$ until the turbidity of 0.5 McFarland standard was achieved.

The comparison was done visually in adequate light against Wickerham card..After achieving the required turbidity sample for AST was taken using a sterile cotton swab dipped into inoculum suspension. Any excess inoculum was removed by rotating and firmly pressing it against the inner wall of the tube above the fluid level. Lawn culture was done over the entire surface of sterile Mueller Hinton agar plate.

Thereafter, antimicrobial discs supplied by HiMedia Laboratories, India, were dispensed evenly onto the surface of inoculated agar plates which were incubated within 15 minutes for $16-18$ hours at $35^{\circ} \mathrm{C}$.Eventually, the results were read and interpreted.After16-18 hours of incubation, plates were viewed with unaided eye using reflected light for the presence or absence of zones of inhibition around the antibiotic discs. Strain of ATCC Enterococcus faecalis 29212 was used as control.

\section{Statistical Analysis}

Microsoft Excel and Microsoft word (version 8.1) were used to generate the tables and figures. Results are based on descriptive statistics.

\section{Results and Discussion}

A total of 13,046 considering exudate and urine samples were received in our laboratory. Among them, 1822 (14\%) showed growth on culture plates. Enterococcus spp. were isolated and identified in 286 isolates, of which $234(83 \%)$ belonged IPD and $52(17 \%)$ to OPD. Speciation was done using the standard laboratory protocol using phenotypic method of identification. Of 286 Enterococcus isolates, $264(92.3 \%)$ were E.faecalis and 22 (7.6\%) were E.faecium (Table 1). Male and female ratio was 1:1.17(Figure1). Age wise distribution showed greater no. of cases in the age group of 40-49(27\%) followed closely by 20-29(20\%) (Table 2). Majority of isolates were recovered from urine (uropathogen) i.e. $246(86 \%)$ (Figure 2).

Isolates were found to be resistant to ciprofloxacin( $92 \%)$, levofloxacin (85\%), high level gentamicin(40\%) and ampicillin(39\%). A single isolate from urine showed resistance to linezolid which was from a patient admitted to ICU. Urinary isolates were moderately sensitive (78\%) to nitrofurantoin. Resistance to vancomycin(VRE) was found in $4 \%$ of urinary samples, isolated from IPD patients.

Enterococci have now emerged as an increasingly important nosocomial pathogen. These infections are recognized by 3 ts tough, tenacious and often times troublesome (Edwards et al., 2000). CDC in a survey on nosocmial diseases, indicated that Enterococci accounted for $13.9 \%$ infections, being next to Escherichia coli(CDC 1989-93). 
Table.1

\begin{tabular}{|c|c|c|}
\hline Specimen & E.faecalis & E.faecium \\
\hline Urine & 228 & 18 \\
\hline Pus & 14 & 02 \\
\hline Ascitic fluid & 08 & 00 \\
\hline Pleural fluid & 02 & 00 \\
\hline HVS & 04 & 00 \\
\hline Conj.swab & 02 & 00 \\
\hline Tissue & 02 & 00 \\
\hline BAL & 04 & 02 \\
\hline
\end{tabular}

Table.2

\begin{tabular}{|c|c|c|c|c|c|c|c|c|c|}
\hline \multicolumn{7}{|c|}{ Nature Of Specimen Recovered From Different Age-Groups } \\
\hline AGE & A/F & BAL & CONJ.SW & HVS & P/F & PUS & TISSUE & URINE & Grand Total \\
\hline $\mathbf{1 0 - 1 9}$ & & & & & & 2 & & 16 & $\mathbf{1 8}$ \\
\hline $\mathbf{2 0 - 2 9}$ & 2 & & & 4 & & & & 52 & $\mathbf{5 6}$ \\
\hline $\mathbf{3 0 - 3 9}$ & 2 & 2 & 2 & & 2 & 4 & & 44 & $\mathbf{5 6}$ \\
\hline $\mathbf{4 0 - 4 9}$ & 4 & 2 & & & & 8 & 2 & 62 & $\mathbf{7 8}$ \\
\hline $\mathbf{5 0 - 5 9}$ & & & & & & & & 32 & $\mathbf{3 2}$ \\
\hline $\mathbf{6 0 - 6 9}$ & & 2 & & & & 2 & & 22 & $\mathbf{2 6}$ \\
\hline $\mathbf{7 0 - 8 0}$ & & & & & & & & 18 & $\mathbf{1 8}$ \\
\hline Grand Total & $\mathbf{8}$ & $\mathbf{6}$ & $\mathbf{2}$ & $\mathbf{4}$ & $\mathbf{2}$ & $\mathbf{1 6}$ & $\mathbf{2}$ & $\mathbf{1 4 6}$ & $\mathbf{2 8 6}$ \\
\hline
\end{tabular}

Fig.1

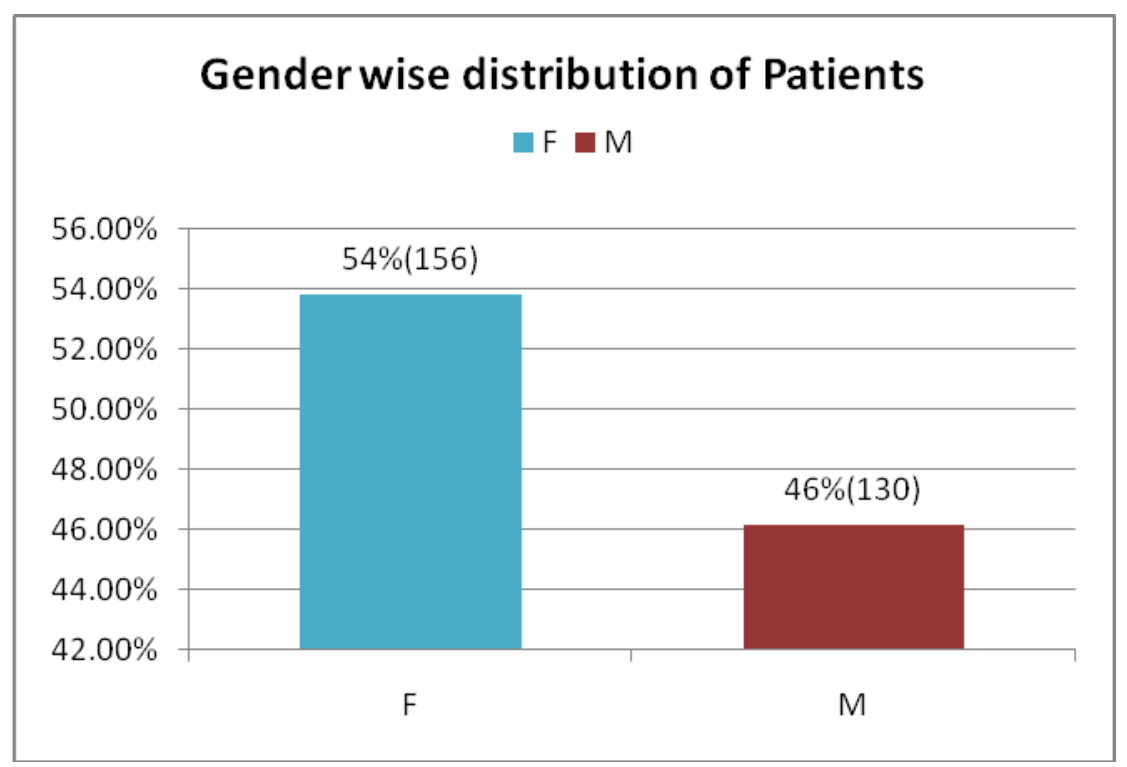


Fig.2

\section{Sample wise Distribution of Enterococci spp.}

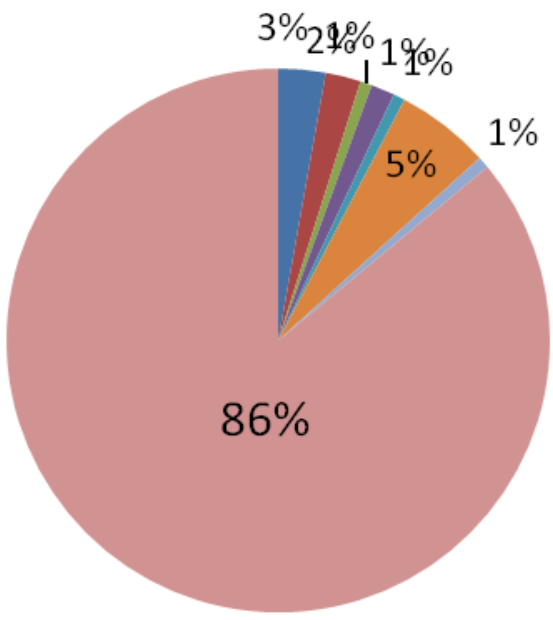

$\mathrm{A} / \mathrm{F}$

BAL

CONJ.SW

- HVS

$\mathrm{P} / \mathrm{F}$

PUS

TISSUE

urine

Fig.3

\section{Comparative Antibiogram of} Enterococci spp.

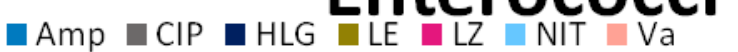

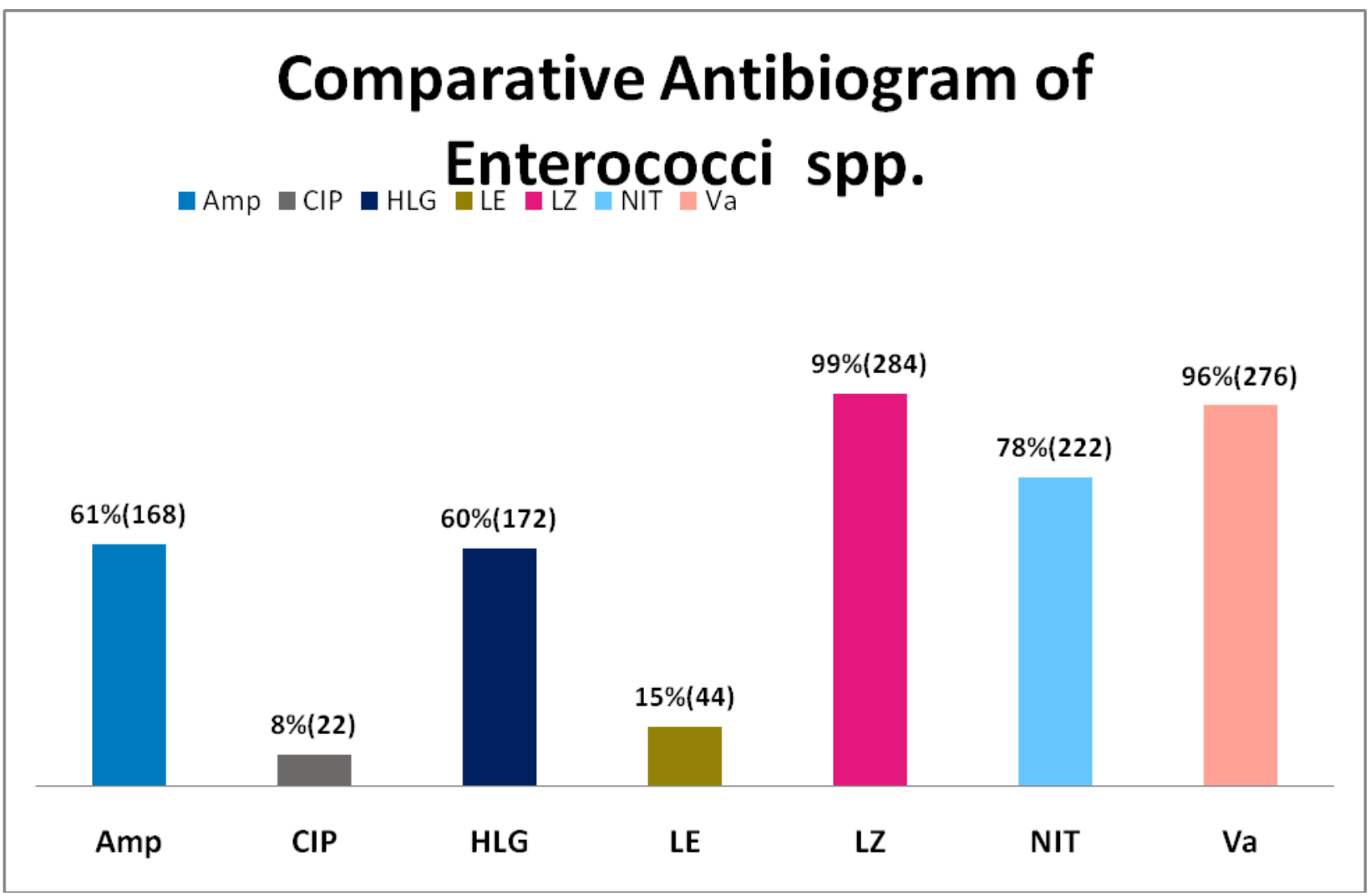


Hence, it is important to know the changing pattern of its antibiogram. Frequently considered to be commensals, Enterococci has come into picture due to their ability to cause infections especially in immunocompromised and hospitalized patients. In our study most of the isolates were recovered from urine followed by pus and blood. Studies done ones estimating the burden of Enterococcal infections support our findings (24). This study also reveals a drastic increase in resistance of the organism to fluoroquinolones which again is comparable to the study conducted in Bangalore (Sreeja et al., 2012). Resistance to ciprofloxacin was found to be $92 \%$. Similar pattern of high resistance to ciprofloxacin was reported by Butch et al., and Subbalaxami et al., (Butcu et al., 2011 \& Subbalaxmi et al., 2010). High resistance to ciprofloxacin probably could be because of its irrational use as over-the-counter drug. Study by Clara Sinel showed that since fluoroquinolones are extensively prescribed, sub-inhibitory concentrations(SICs)are likely to occur in vivo, with potential effects on bacterial metabolism with subsequent modulation of opportunistic traits (Sinel et al.,2017).

Resistance of our urinary isolates against nitrofurantoin was $22 \%$ which is in concordance with other studies(Kaur et al., 2003 \& Meena et al., 2017).

The drug of choice for serious Enterococcal infections is an aminoglycoside in combination with a cell wall active agent. However, high-level aminoglycoside resistance (HLAR) is responsible for loss of synergy between cell wall active agents and aminoglycoside (Bekhit et al., 2011). Studies conducted in various countries also report varying rates of resistance to HLG. A study from India( Shah et al., 2012) reported 40\%, $53 \%, 68 \%$, and $8 \%$ resistance rates for ampicillin, high-level gentamicin, high-level streptomycin, and vancomycin respectively. Another study done in 2007 by Gupta et al., reported $75 \%$ resistance rate for high-level gentamicin. In our study, resistance to HLG was found to be around $40 \%$ similar to study by Mendiratta et al.,(10).

Most recently, there has been reports of rising rates of resistance in Enterococci especially to Vancomycin (Chaudhary et al., 2007 \& Devi et al.,2002). In our study $4 \%$ isolates were VRE, which shows significant similarity to results (1.7-20\%) from studies done in other parts of India(9-11). Study at Apollo hospitals Chennai(2012) showed similar findings with prevalence of VRE of about 4\%(Vidyalakshmi et al.,2012).Research work from Southern part of India revealed higher percentage of VRE (Parmeswarappa et al., 2013 \& Karmarkar et al., 2004). Emergence of VRE has been attributed to the indiscriminate use of vancomycin, the colonization pressure and noncompliance with the infection control measures(Tripathi et al., 2016,Vidyalakshmi et al., 2012, Biswas et al., 2016)

Antibiotic resistance is an ever-increasing problem complicating therapy in patients with bacterial infections, especially Enterococcal. They pose a major therapeutic challenge because of having both intrinsic and acquired resistance to various antibiotics. Widespread use, misuse and easy availability of these antibiotics over the counter without any prescription is considered to play a major contributory role. Change in pattern of antibiotic resistance in Enterococcus spp., with the emergence of vancomycin resistance (VRE) and high-level aminoglycoside resistance is of major concern. Currently, specific recommendation for antimicrobial prescription of VRE is lacking. Moreover, at most of the clinical settings, the protocol for follow-up surveillance is inadequate (Addisu et al., 2020). Increased prevalence of MDR Enterococci constitute a major health issue, 
leading to high rates of morbidity and mortality, economic loss and limited treatment options. To encounter this alarmingly increasing health problem, there is urgent need for evidence-based research, focusing on the identification of the factors facilitating the transmission of Enterococcal antimicrobial resistance within the hospital environment. Prudent use of antibiotics is a fundamental strategy to deal with the problem of antibiotic resistance.

\section{References}

Addisu Melese, Chalachew Genet \& Tesfaye Andualem Prevalence of Vancomycin resistant enterococci (VRE) in Ethiopia: a systematic review and meta-analysis BMC Infectious Diseases volume 20, Article number: 124 (2020)

Arias, C. A. and B. E. Murray, "The rise of the Enterococcus beyond vancomycin resistance," Nature Reviews Microbiology, vol. 10, no. 4, pp. 266-278, 2012.

Bhatt, P., A. Patel, A. K. Sahni et al., "Emergence of multidrug resistant enterococci at a tertiary care centre," Medical Journal Armed Forces India, vol. 71, no. 2, pp. 139-144, 2015.

Biswas, P., S. Dey, L. Adhikari, and A. Sen, "Detection of vancomycin resistance in enterococcus species isolated from clinical samples and feces of colonized patients by phenotypic and genotypic methods," Indian Journal of Pathology and Microbiology, vol. 59, no. 2, pp. 188-193, 2016.

Butcu M, Akcay SS, Inan AS, Aksaray S, Engin S, \& Calisici G. In vitro susceptibility of enterococci strains isolated from urine samples. J Infect chemother. 2011 Aug;17(4):575-8.

Centers for Disease Control and Prevention. 1993. Nosocomial enterococci resistant to vancomycin-United States, 1989-1993. Morb. Mortal. Wkly. Rep. 42:597-599.

Chakraborty, N. Pal, S. Sarkar, and M. Gupta, "Antibiotic resistance pattern of Enterococci isolates from nosocomial infections in a tertiary care hospital in Eastern India," Journal of Natural Science, Biology and Medicine, vol. 6, no. 2,pp. 394-397, 2015.

Chaudhary U, Shamma M, Yadav A. The antimicrobial susceptibility patterns of the common and the unusual Enterococcus species which were isolated from clinical specimens. J Infect Dis Antimicrobial Agents. 2007;24:55-62.

Clinical and Laboratory Standards Institute (CLSI), Performance Standards for Antimicrobial Susceptibility Testing Wayne, Pennyslvania, USA, 26th edition, 2016.

Devi P S, Rao S P, Shivananda P G. The characterization, antibiotic susceptibility pattern and the detection of betalactamases in Enterococci. Indian $\mathrm{J}$ of PatholMcrobiol. 2002;45:79-82.

DK Mendiratta, H Kaur, V Deotale, D C Thamke, R Narang, P Narang "Status of high level aminoglycoside resistant Enterococcus faecium and Enterococcus faecalis in arural hospital of central India" Indian Journal Of Medical Microbiology.e0276316

Edwards DD. Enterococci attract attention of concerned microbiologists. ASM News $2000 ; 66: 540$

Facklam, R. R. and M. D. Collins, "Identification of Enterococcus species isolated from human infections by a conventional test scheme," Journal of Clinical Microbiology, vol. 27, no. 4, pp. 731-734, 1989.

Gupta V, Singla N. Speciation and Antimicrobial Susceptibility pattern of Enterococci from a Tertiary Health Care Center of North India. J ClinDiag Res 2007; 5: 385- 389

Karmarkar MG, Gershom SE, Mehta PR. The Enterococcal infections with a special reference to the phenotypic characterization and the drug resistance. Indian J Med Res. 2004;119:22-25.

Kaur N, Sharma S, Malhotra S, Madan P, Hans C. Urinary tract infection:Aetiology and antimicrobial resistance pattern in infants from a tertiary care hospital in northern India. J ClinDiagn Res. 2014;8:DC01-03. 
Meena S, Mohapatra S, Sood S, Dhawan B, Das BK, Kapil A. Revisiting nitrofurantoin for vancomycin resistant enterococci. J Clin Diagn Res 2017;11:DC19- DC.

Monica Garcia Solacha, Louis B.Rice. The Enterococcus:A model of Adaptibility to its Environment $\mathrm{J}$ of Clinical Microbiology Reviews,ASM,2019.

Mulla S, Patel KG, Panwala T, Rewadiwala S, The prevalence of Enterococci with a higher resistance level in a tertiary care hospital: A matter of concern. National J Med Res 2012 2(1):25-27.

Murray, B. E., Vancomycin-resistant enterococcal infections, The New England Journal of Medicine, vol. 342, no. 10, pp. 710-721, 2000.

Murray, P. R, E. J. Baran, M. A. Pfaller, and R. Y. Tenover, "Manual of clinical microbiology 8th edition," in Special Tests

Parameswarappa, J., V. P. Basavaraj, and C. M. Basavaraj, "Isolation, identification, and antibiogram of Enterococci isolated from patients with urinary tract infection," Annals of African Medicine, vol. 12, no. 3, pp. 176-181, 2013.

Salem-Bekhit, M. M., I. M. Ibrahim Moussa, M. M. Muharram, A. M. Elsherbini, and S. AlRejaie, "Increasing prevalence of highlevel gentamicin resistant enterococci: an emerging clinical problem," African Journal of Microbiology Research vol. 5, no. 31, pp. 5713-5720, 2011.

Shah L, Mulla S, Patel KG, Rewadiwala S. Prevelance of enterococci with higher resistance level in a tertiary care hospital: A matter of concern. Nat J Med Res 2012;

Sinel, C., M. Cacaci, P. Meignen, et al.,
Subinhibitory concentrations of ciprofloxacin enhance antimicrobial resistance and pathogenicity of Enterococcus faecium Antimicrob Agents Chemother, 61 (2017) e02763-16.

Sreeja, S., P. R. Sreenivasa Babu, and A. G. Prathab, "prevalence and the characterization of the enterococcus species from various clinical samples in a tertiary care hospital, "Journal of Clinical and Diagnostic Research: JCDR, vol. 6,no. 9, pp. 1486-1488, 2012.

Subbalaxmi MVS, Lakshmi V, Lavanya V. Antibiotic resistance - experience in a tertiary care hospital in South India. Journal of Association of Physicians of India. Dec.2010; 58: 18-22.

Tripathi, S. K. Shukla, A. Singh, and K. N. Prasad, "Prevalence, outcome and risk factor associated with vancomycin-resistant Enterococcus faecalis and Enterococcus faecium at a Tertiary Care Hospital in Northern India," Indian Journal of Medical Microbiology, vol. 34, no. 1, pp. 38-45,2016.

Vidyalakshmi P R, Gopalakrishnan R, Ramasubramanian V, Ghafur A K, Nambi P S, Thirunarayana 23MA. The clinical, epidemiological, and the microbiological profiles of the patients with vancomycinresistant Enterococci from a tertiary care hospital. J Global Infect Dis. 2012;4:13738.

Winn, W. C., Koneman's Color Atlas and Textbook of Diagnostic Microbiology, Lippincott Williams \& Wilkins, Philadelphia, PA, USA, 2006.

\section{How to cite this article:}

Shiwani Mishra, Richa Sinha, Namrata Kumari, Kumar Saurabh, Shailesh Kumar and Shahi, S. K. 2021. Prevalence and Antibiotic Susceptibility Pattern of Enterococcus spp. from Various Clinical Samples of Patients at A Tertiary Care Hospital, Patna. Int.J.Curr.Microbiol.App.Sci. 10(04): 163-170. doi: https://doi.org/10.20546/ijcmas.2021.1004.015 